\title{
Porphyrin-based metal coordination polymers with self-assembly pathway-dependent properties for photodynamic and photothermal therapy
}

\section{Yuyang Miao}

Shandong University

Shibo Lv

Shandong University

Daoyuan Zheng

Shandong University

Yuhan Liu

Shandong University

Dapeng Liu ( $\nabla$ dapengliu@sdu.edu.cn )

Shandong University

\section{Fengling Song}

Shandong University

\section{Article}

Keywords: Porphyrin-based metal coordination polymers, MCPs, phototherapy application, self-assembly process

Posted Date: August 12th, 2020

DOl: https://doi.org/10.21203/rs.3.rs-54280/v1

License: (c) (i) This work is licensed under a Creative Commons Attribution 4.0 International License.

Read Full License

Version of Record: A version of this preprint was published at Biomaterials Science on January 1st, 2021. See the published version at https://doi.org/10.1039/D0BM02112A. 


\section{Abstract}

Porphyrin-based metal coordination polymers (MCPs) have attracted numerous attention due to their great promise application in phototherapy including photodynamic therapy (PDT) and photothermal therapy (PTT). However, the detailed self-assembly process of porphyrin-based MCPs still remains poorly understood. This work provides a detailed study of the self-assembly process of MCPs constructed by Mn2+ and TCPP (TCPP: 4,4',4",4"'-(Porphine-5,10,15,20-tetrayl)tetrakis(benzoic acid)) in aqueous solution. Unlike traditional nucleation and growth mechanism, we discover that there is a metastable metal-organic intermediate which is kinetically favored in the self-assembly process. And the metastable metal-organic intermediate nanotape structures could convert into thermodynamically favored nanosheets through disassembling into monomers followed by reassembling process. Moreover, the two structurally different assemblies exhibit distinct photophysical performances. The intermediate Mn-TCPP aggregates show good light-induced singlet oxygen 102 generation for PDT while the thermodynamic favored stable Mn-TCPP aggregates exhibit good photothermal conversion ability as photothermal agents (PTAs). This study could facilitate controlling self-assembly pathway to fabricate complex MCPs with desirable applications.

\section{Introduction}

Metal coordination polymers (MCPs) are constructed from coordination metal ions and organic linkers followed by supramolecular assembly with hydrogen bonding, $\pi-\pi$ stacking or hydrophobic interactions. ${ }^{1-5}$ Owing to their facile synthetic procedure, high drug loading capability and low long-term toxicity, the MCPs have attracted numerous interest in biomedical application. ${ }^{6-10}$ In the past decades, large numbers of metal-organic nanomaterials for anticancer therapy were fabricated with different sizes and morphologies. ${ }^{4,11-12}$ Among the reported MCPs, porphyrin-based MCPs are widely studied. ${ }^{13-16}$ Porphyrins are well-known photosensitizers for photodynamic therapy due to their highly efficient singlet oxygen ${ }^{1} \mathrm{O}_{2}$ generation under light irradiation. ${ }^{16-17}$ Moreover, Zheng et, al reported that porphyrins could possess photothermal conversion capability when porphyrins aggregated with high packing density. ${ }^{18-19}$ So, porphyrins could also be utilized as photothermal agents (PTAs) for photothermal therapy (PTT). These dual functionalities endow porphyrin great promise in phototherapy. Moreover, in the MCPs, porphyrins directly participate in constructing the structure of MCPs, which makes MCPs have relatively high photosensitizer loading efficiency. ${ }^{14,20}$ At last, the metal ions such as $\mathrm{Mn}^{2+}, \mathrm{Gd}^{3+}$ offer magnetic resonance imaging (MRI) which endows the formed MCPs with bioimaging applications. ${ }^{21-22}$ All these merits make the porphyrin-based MCPs provide great therapeutic efficiency in precise anticancer therapy. Although much attention has been paid to the fabrication of porphyrin-based MCPs with various morphologies and functions, the deep understanding of the self-assembly mechanism which controlled the final nanostructures remained elusive.$^{23-25}$ The kinetic detailed study was rarely reported but crucial in view of controlling the self-assembly pathway to fabricate the metal-organic nanoparticles with desired applications. ${ }^{23-24,26-27}$ And the corresponding formed nanostructures usually exhibited unique properties such as diversity, adaptivity and complexity. ${ }^{28}$ 
In this paper, we studied the self-assembly process of Mn-TCPP (TCPP: 4,4',4",4"'-(Porphine-5,10,15,20tetrayl)tetrakis(benzoic acid)) nanoparticles (NPs) (Scheme 1). Interestingly, we discovered that during the self-assembly process, kinetically formed metastable amorphous Mn-TCPP intermediates (tape structures) were first formed before converting into thermodynamically favored form. The kinetically formed metastable Mn-TCPP aggregates exhibited different morphologies and photophysical properties from the thermodynamic stable states. We also found that the metastable Mn-TCPP aggregates converted into thermodynamic stable assemblies (nanosheets) via disassembling into monomers followed by the reassembling process. Moreover, the different photophysical properties endowed the MnTCPP aggregates different phototherapy applications. The kinetically formed metastable Mn-TCPP aggregates showed efficient photoinduced singlet oxygen generation $\left({ }^{1} \mathrm{O}_{2}\right)$ for PDT application. Meanwhile, the thermodynamic stable Mn-TCPP aggregates exhibited photothermal conversion ability as photothermal agents (PTAs). This research provided a new insight of the formation of porphyrin-based MCPs for phototherapy including PDT and PTT.

\section{Results And Discussion}

\section{Kinetic study of the self-assembly behavior of Mn-TCPP assemblies}

\section{Self-assembly pathway of Mn-TCPP assemblies}

In aqueous solution, $\mathrm{Mn}^{2+}$ and TCPP were mixed in aqueous solution under vigorous stirring followed by slow addition of $\mathrm{OH}^{-}$at $328 \mathrm{~K}$ to initiate self-assembly (Figure 1a). After reaction for ca. $1 \mathrm{~h}, \mathrm{Mn}$-TCPP colloid was formed, which was verified by Tyndall effect under laser irradiation (Figure S1a). The size and morphology of the formed Mn-TCPP assemblies were characterized by transmission electron microscope (TEM) and dynamic light scattering (DLS). As shown in Figure S1b, nanoparticles with ca.10 $\mathrm{nm}$ were clearly seen in the TEM images. DLS profile (Figure S1b) showed that the hydrodynamic diameter $\left(D_{h}\right)$ of the Mn-TCPP assemblies was $20 \mathrm{~nm}$ with PDI 0.196 which indicated there were some degrees of aggregation. Atomic force microscope (AFM) images confirmed the height of the nanosheets was about $5 \mathrm{~nm}$ (Figure S2a) which suggested nanosheets were formed. The nanosheet structures were further verified by scanning electron microscope (SEM) images shown in Figure S2b. Moreover, energy dispersive spectrometer (EDS) and X-ray photoelectron spectroscopy (XPS) profiles (Figure S3 and Figure S4) confirmed the constituent of the Mn-TCPP nanosheets consisted of $\mathrm{Mn}$ and $\mathrm{N}$ element. The components of the as-prepared Mn-TCPP nanosheets were also confirmed by TG analysis (Figure S5). TG analysis demonstrated that the Mn content in the Mn-TCPP nanosheets was about $14 \mathrm{wt} \%$.

Interestingly, during the formation of the Mn-TCPP colloid, we found the solution color changed from yellow to pink before the colloid transformed to final brown as shown in the insert image of Figure 1c. UV/Vis spectrum was employed to measure the three different states. As shown in Figure 1c, the maximum absorption peak was $435 \mathrm{~nm}$ (solution color: yellow) shifted to $415 \mathrm{~nm}$ (solution color: pink) 
with gradient addition of $\mathrm{OH}^{-}$. With further addition of $\mathrm{OH}^{-}$, the solution color changed from yellow to brown accompanied redshift of the maximum absorption peak from $415 \mathrm{~nm}$ to $465 \mathrm{~nm}$. Here we could see that during the self-assembly process of Mn-TCPP nanosheets, there were three different energy states formed: initial state, intermediate state and thermodynamically favored state. This interesting phenomenon encouraged us to attempt to study the self-assembly pathway of Mn-TCPP assemblies. UV/Vis spectra were further used to monitoring changes in the absorption spectrum from an initial state to an intermediate state, then to thermodynamically favored state. The absorption intensity changes at $465 \mathrm{~nm}$ as a function of $\mathrm{OH}^{-}$amount exhibited an obvious non-isodesmic model where there was a lag seen in the initial process (Figure 1d). ${ }^{29}$ This kinetic profile was characteristic of a cooperative process. ${ }^{30}$ In addition, the self-assembly process was temperature-dependent which was verified by temperaturedependent kinetic profiles (Figure S6). Here we could see that the self-assembly pathway could be divided into three different phases. We referred to the pathway from the initial state to the intermediate state as phase I and the pathway from the intermediate state to the thermodynamically favored state was named as phase II. The thermodynamic stable state was referred as phase III.

In the phase $\mathrm{I}$, addition of $\mathrm{OH}^{-}$quickly induced the decrease of the peak $(435 \mathrm{~nm})$ while the peak at 415 $\mathrm{nm}$ increased sharply as shown in Figure 2a. The corresponding plot of $\mathrm{I}_{415} / \mathrm{I}_{435}$ as a function of $\mathrm{OH}^{-}$ amount was shown in Figure $2 \mathrm{~b}$. The plot could be fitted to a first-order kinetic model. This $\mathrm{I}_{415} / \mathrm{I}_{435}$ change could be ascribed to the formation of TCPP-COO', which was confirmed by the control experiment where addition of $\mathrm{OH}^{-}$into the TCPP solution without $\mathrm{Mn}^{2+}$. In the control experiment, a similar trend in $\mathrm{I}_{415}$ and $\mathrm{I}_{435}$ was observed (Figure 2c). However, by comparing the two plots of $\mathrm{I}_{415} / \mathrm{I}_{435}$ versus $\mathrm{OH}^{-}$ amount, we could see that there was a big gap shadowed in Figure $2 \mathrm{~b}$. This gap indicated that $\mathrm{OH}^{-}$could be consumed in another form in the Mn/TCPP solution. This view was supported by the formation of $\mathrm{Mn}(\mathrm{OH})_{2}$ (Figure S7a) when $\mathrm{Mn}^{2+}$ and TCPP were mixed in a higher molar ratio (TCPP: $\mathrm{Mn}^{2+}$ ) of 1:10 followed by addition of $\mathrm{OH}^{-}$. This experiment suggested that TCPP and $\mathrm{Mn}^{2+}$ both consumed $\mathrm{OH}^{-}$in a competitive manner. Moreover, with the further addition of $\mathrm{OH}^{-}$, no absorption peak at $465 \mathrm{~nm}$ was observed in the control experiment (Figure 2c), which suggested that $\mathrm{Mn}^{2+}$ was crucial in the formation of thermodynamic stable Mn-TCPP aggregates. The formation of - $\mathrm{COO}^{-}$in TCPP could provoke the coordination between $\mathrm{Mn}^{2+}$ and TCPP. This was verified by the shift of $-\mathrm{C}=0$ stretching peak in the IR spectrum (Figure S7b). The formed intermediate Mn-TCPP aggregates were characterized by TEM, EDS, XPS and XRD. As shown in Figure $2 \mathrm{~d}$ and Figure S8a, the Mn-TCPP aggregates revealed one-dimensional assemblies. The height profile in the AFM image (Figure S8b) indicated that the height of Mn-TCPP assemblies was about $2 \mathrm{~nm}$, which suggested the one-dimensional Mn-TCPP assemblies were tape structures. The EDS and XPS profiles (Figure S9 and Figure S10) confirmed the formed intermediate MnTCPP tape structures constituted Mn and N elements. And TG analysis (Figure S11) demonstrated that the content of $\mathrm{Mn}$ in the intermediate Mn-TCPP structures was as high as $55 \%$ which was similar to the results obtained from ICP-AAS (Mn content : $54 \mathrm{wt} \%$ ). This confirmed that the high content of $\mathrm{Mn}$ in the intermediate Mn-TCPP aggregates. XRD profile verified that the formed intermediate Mn-TCPP tape structures were amorphous structures (Figure S12). Moreover, from UV-Vis spectra, we could see that the 
maximum absorption wavelength was at $415 \mathrm{~nm}$, which indicated the TCPP was in a monomer state in the formed intermediate Mn-TCPP aggregates. ${ }^{31}$ According to the above data, we could see that the intermediate Mn-TCPP tape structures could consist of large amounts of $\mathrm{Mn}(\mathrm{OH})_{2}$ and few $\mathrm{Mn}-\mathrm{TCPP}$ complexes where the Mn-TCPP complexes separately anchored in the amorphous $\mathrm{Mn}(\mathrm{OH})_{2}$ complexes.

From the above experiments, we could summary the possible self-assembly pathway in the phase I. Addition of $\mathrm{OH}^{-}$could induce the formation of - $\mathrm{COO}^{-}$in TCPP and large amounts of $\mathrm{Mn}(\mathrm{OH})_{2}$ in a competitive manner. Subsequently, Mn-TCPP complexes formed by coordination between $\mathrm{Mn}^{2+}$ and TCPP ( $\left.\mathrm{COO}^{-}\right)$anchored into the amorphous $\mathrm{Mn}(\mathrm{OH})_{2}$ aggregates in a single monomer state.

However, the formed intermediate Mn-TCPP aggregates were kinetically formed state and not stable, which could further transform to thermodynamically favored state. The transformation from kinetically formed state to thermodynamically favored state was investigated by UV/Vis spectra. As shown in Figure $3 a$, continue addition of $\mathrm{OH}^{-}$could decrease the absorption intensity at $415 \mathrm{~nm}\left(\mathrm{I}_{415}\right)$ with $\mathrm{I}_{465}$ increasing. The change of $\mathrm{I}_{415} / \mathrm{I}_{465}$ versus additional $\mathrm{OH}^{-}$amount was seen in Figure S13. $\mathrm{I}_{415} / \mathrm{I}_{465}$ decreased as a function of $\mathrm{OH}^{-}$amount in an exponential decay mode. Moreover, the decay rate was temperaturedependent which was verified by comparing plots under $308 \mathrm{~K}$ and $328 \mathrm{~K}$ (Figure S13). Interestingly, even without addition of $\mathrm{OH}^{-}$, the transformation from kinetic favored Mn-TCPP assemblies to thermodynamically favored Mn-TCPP assemblies with time was observed seen in Figure 3b. As time going, the $\mathrm{I}_{415} / \mathrm{I}_{465}$ decreased sharply and quickly achieved a thermodynamically favored state in $0.5 \mathrm{~h}$ at $328 \mathrm{~K}$. The transformation rate was temperature-dependent seen in Figure 3b. decreasing temperature could significantly decline the transformation rate. For example, when the temperature decreased to 318 $\mathrm{K}$, it needed $c a .1 \mathrm{~h}$ to achieve the thermodynamic equilibrium state shown in Figure $3 \mathrm{~b}$. In addition, the transformation rate was also dependent on solution conditions. We centrifuged the intermediate MnTCPP assemblies and monitored the $\mathrm{I}_{415} / \mathrm{I}_{435}$ change with time in the deionized water instead of the original solution (Figure $3 \mathrm{c}$ ). In this case, the transformation from intermediate Mn-TCPP assemblies to thermodynamic equilibrium state needed much longer time (more than $6 \mathrm{~h}$ ) at $328 \mathrm{~K}$. Figure $3 \mathrm{c}$ revealed that in deionized water, increasing temperature could accelerate the transformation of intermediate MnTCPP aggregates as well. By comparing the transformation rates of intermediate Mn-TCPP aggregates in original solutions and deionized water, we hypothesized that the transformation from Mn-TCPP intermediates to the thermodynamically favored state could be related to TCPP monomers. To verify this view, we added TCPP/OH ${ }^{-}$into the intermediate TCPP-Mn assemblies in water. From the plot of absorption intensity at $465 \mathrm{~nm}$ versus time (Figure S14), we could clearly see that adding TCPP/OH could accelerate the transformation process, which indicated that TCPP monomer involved in the transformation process. We also did the dialysis experiment of intermediate Mn-TCPP assemblies against water with a dialysis bag (molecular cut-off value: 3500 ). The solution outside of the dialysis bag was monitored by UV. After $14 \mathrm{~h}$, the absorption peak at $415 \mathrm{~nm}$ was detected which was corresponding to the phase I process (Figure 3d). After 3 days, the absorption intensity at $465 \mathrm{~nm}$ began to increase which suggested the phase II occurred. From these experiments, we could see that in the phase II, the 
intermediate Mn-TCPP tape structures first disassembled into monomer TCPP and $\mathrm{Mn}^{2+}$ followed by reassembling into thermodynamically favored Mn-TCPP nanosheets.

\section{Photophysical properties of Mn-TCPP assemblies}

The photophysical properties of Mn-TCPP assemblies in different states were investigated. The absorption profiles (Figure 1c) of Mn-TCPP assemblies in different states have been discussed above. And the various absorption behaviors of Mn-TCPP assemblies were resulted from the aggregation types of TCPP ligands. Here Figure 4a compared fluorescence of the Mn-TCPP assemblies in different states. From Figure 4a, we could find that the fluorescence of the thermodynamic stable Mn-TCPP nanosheets was completely quenched, while the intermediate Mn-TCPP aggregates still exhibited relatively strong fluorescence.

Meanwhile, as shown in nanosecond time-resolved transient absorption spectra (NTAS) (Figure 4b), a significant feature of transient absorption was observed at $460 \mathrm{~nm}$ under $355 \mathrm{~nm}$ laser excitation, which suggested that there was a triplet excited signal for intermediate Mn-TCPP tape structures. This could be attributed to the monomer state of porphyrin in the intermediate structures. However, under the same experimental conditions, no triplet excited signal was detected for thermodynamic stable Mn-TCPP nanosheets (Figure S15). No FL emission and triplet state in the thermodynamic stable Mn-TCPP nanosheets could be attributed to the $\mathrm{J}$-aggregate induced quenching effect of the porphyrin moieties. ${ }^{18,32}$ These different photophysical properties between intermediate Mn-TCPP assemblies and thermodynamic stable Mn-TCPP assemblies demonstrated that the metal-organic nanomaterials could exhibit self-assembly pathway-dependent properties.

\section{In vitro PDT/PTT experiment}

The different photophysical properties endowed these two Mn-TCPP aggregates with different applications. In terms of the intermediate state, the triplet signal detected by NATS demonstrated that the intermediate Mn-TCPP assemblies could possess light-induced ${ }^{1} \mathrm{O}_{2}$ generation capability as PDT photosensitizers. And the thermodynamic favored aggregates with no FL emission could convert light energy into heat for PTT. Based on this hypothesis, we first evaluate the photoinduced singlet oxygen generation ability of the intermediate Mn-TCPP aggregates with 1,3-diphenylisobenzofuran (DPBF) as a probe. The DPBF was widely used as a ${ }^{1} \mathrm{O}_{2}$ sensor due to its decreasing absorbance intensity at approximately $410 \mathrm{~nm}$ when exposure to $\mathrm{O}_{2}$ (Figure $5 \mathrm{a}$ ). Upon light irradiation $\left(660 \mathrm{~nm}, 12 \mathrm{~mW} \mathrm{~cm} \mathrm{~cm}^{-2}\right.$ ) within 20 min, negligible changes were noted in the DPBF control group. However, a continuous decrease of the DPBF absorption at $410 \mathrm{~nm}$ was both observed in the two Mn-TCPP aggregates samples, which suggested their ${ }^{1} \mathrm{O}_{2}$ generation ability. Moreover, for the intermediate Mn-TCPP aggregates, a significant decrease was clearly noted, suggesting the highly efficient light-induced ${ }^{1} \mathrm{O}_{2}$ generation ability. This experiment demonstrated that the intermediate Mn-TCPP aggregates possessed excellent ${ }^{1} \mathrm{O}_{2}$ generation ability for PDT. 
To verify the photothermal property of the Mn-TCPP aggregates, we recorded the temperature changes of the two Mn-TCPP aggregates samples with an infrared (IR) thermal mapping apparatus. And the PBS solution was served as a control. As shown in Figure $5 b$, under laser irradiation at a low laser power density of $200 \mathrm{~mW} \mathrm{~cm}^{-2}$ within 10 minutes, the temperature in the thermodynamic favored aggregates increased from $24^{\circ} \mathrm{C}$ to $41^{\circ} \mathrm{C}$ maximumly, which exhibited good photothermal conversion ability. And the photothermal conversion behavior of the thermodynamic favored aggregates were further explored in various laser-irradiation time and concentrations (Figure S16a and Figure S16b), suggesting that the photothermal behavior was concentration-and laser-irradiation time dependent. At last, the photostability of the thermodynamic stable aggregates were explored, As shown in Figure S16c, when exposed to a laser for $24 \mathrm{~min}$, negligible change in the maximum temperature was noted in the thermodynamic stable Mn-TCPP aggregates sample. This suggested that the thermodynamic stable Mn-TCPP aggregates exhibited excellent photothermal stability. Here the good photothermal conversion capability of the thermodynamic stable Mn-TCPP aggregates could be caused by strong $\pi-\pi$ stacking proposed by Zheng et al. ${ }^{32}$ Thus, the thermodynamic stable Mn-TCPP aggregates could be promising photothermal agents (PTAs) for PTT.

The effects of PDT or PTT on the cell viability were further investigated towards HeLa cells. We first assessed the intracellular ${ }^{1} \mathrm{O}_{2}$ generation by the intermediate Mn-TCPP aggregates after internalization. The dichlorofluorescein diacetate (DCFH-DA) was selected as a ${ }^{1} \mathrm{O}_{2}$ sensitizer, which could convert DCFHDA into highly fluorescent dichlorofluorescein (DCF) and then detected using monitored using confocal laser scanning microscopy (CLSM). As shown in Figure 5c, upon light irradiation, green fluorescence was observed in the cells cultured with intermediate Mn-TCPP aggregates, suggesting good ${ }^{1} \mathrm{O}_{2}$ generation of intermediate Mn-TCPP aggregates in cells. Moreover, from a 3-(4,5-dimethylthiazol-2-yl)-2,5diphenyltetrazolium bromide (MTT) assay (Figure $5 \mathrm{~d}$ and Figure 5e), owing to the contribution of ${ }^{1} \mathrm{O}_{2}$ or photothermal ability of different Mn-TCPP aggregates, an obvious phototoxicity towards HeLa cells was observed. The $50 \%$ growth inhibitory concentration $\left(\mathrm{IC}_{50}\right)$ values against HeLa cells under light irradiation were measured to be $c a .50 \mu \mathrm{g} / \mathrm{mL}$ for intermediate Mn-TCPP aggregates and ca. $0.73 \mathrm{mg} / \mathrm{mL}$ for thermodynamic stable Mn-TCPP aggregates.This vitro toxicity studies demonstrated that the formed MnTCPP aggregates controlled by self-assembly pathway exhibited good antitumor efficiency via PDT or PTT. This suggested that the Mn-TCPP aggregates showed self-assembly pathway-dependent anticancer therapy.

\section{Summary}

In this study, we systematically investigated the self-assembly process of porphyrin-based MCPs based on Mn and TCPP. The metastable "off-pathway" Mn-TCPP aggregates during the self-assembly process were obtained. And the transformation from the intermediate Mn-TCPP aggregates into thermodynamic stable Mn-TCPP assemblies via a disassembling and reassembling process were further confirmed. Moreover, the two aggregates in the self-assembly process exhibited different structures and photophysical properties. Owing to their different photophysical behaviors, the intermediate Mn-TCPP 
aggregates show efficient ${ }^{1} \mathrm{O}_{2}$ generation under light irradiation while the thermodynamic stable $\mathrm{Mn}$ TCPP assemblies exhibit great photothermal conversion capability. This suggested that the porphyrinbased MCPs dictated by the self-assembly pathway could exhibit different functionalities for phototherapy. Our findings provide a significant progress in the designing of porphyrin-based nanomaterials with desired biomedical applications.

\section{Declarations}

\section{Acknowledgments}

We thank Xiaoju Li, Haiyan Yu from the State Key Laboratory of Microbial Technology of Shandong University for help and guidance in TEM and CLSM imaging. We also thank Dr. Guoqing Ren for discussions about XRD and XPS spectra. The research is supported by the Fundamental Research Funds of Shandong University (10000079614034), Guangdong basic and applied basic research foundation (2019A1515110441) and the Key R\&D Program of Shandong Province (2019GSF108021).

\section{Corresponding author}

*Dapeng Liuｄapengliu@sdu.edu.cn

*Fengling Song songfl@sdu.edu.cn

\section{Conflicts of interest}

The authors declare that they have no known competing financial interests.

\section{Author information}

\# The authors contributed equally.

\section{References}

1. Spokoyny, A. M.; Kim, D.; Sumrein, A.; Mirkin, C. A., Infinite coordination polymer nano- and microparticle structures. Chem. Soc. Rev. 2009, 38 (5), 1218-1227.

2. Wang, S.; McGuirk, C. M.; d'Aquino, A.; Mason, J. A.; Mirkin, C. A., Metal-Organic Framework Nanoparticles. Adv. Mater. 2018, 30 (37), 1800202.

3. Novio, F.; Simmchen, J.; Vázquez-Mera, N.; Amorín-Ferré, L.; Ruiz-Molina, D., Coordination polymer nanoparticles in medicine. Coord. Chem. Rev. 2013, 257 (19), 2839-2847.

4. Wang, J.-L.; Wang, X.-Y.; Wang, Y.-H.; Hu, X.-Y.; Lian, J.-R.; Guan, Y.-L.; Chen, H.-Y.; He, Y.-J.; Wang, H.-S., Room-temperature preparation of coordination polymers for biomedicine. Coord. Chem. Rev. 2020, $411,213256$. 
5. Zhu, W.; Zhao, J.; Chen, Q.; Liu, Z., Nanoscale metal-organic frameworks and coordination polymers as theranostic platforms for cancer treatment. Coord. Chem. Rev. 2019, 398, 113009.

6. Horcajada, P.; Chalati, T.; Serre, C.; Gillet, B.; Sebrie, C.; Baati, T.; Eubank, J. F.; Heurtaux, D.; Clayette, P.; Kreuz, C.; Chang, J.-S.; Hwang, Y. K.; Marsaud, V.; Bories, P.-N.; Cynober, L.; Gil, S.; Férey, G.; Couvreur, P.; Gref, R., Porous metal-organic-framework nanoscale carriers as a potential platform for drug delivery and imaging. Nat. Mater. 2010, 9 (2), 172-178.

7. Kitagawa, S.; Kitaura, R.; Noro, S.-i., Functional Porous Coordination Polymers. Angew. Chem. Int. Ed. 2004, 43 (18), 2334-2375.

8. Zhang, P.; Wang, J.; Chen, H.; Zhao, L.; Chen, B.; Chu, C.; Liu, H.; Qin, Z.; Liu, J.; Tan, Y.; Chen, X.; Liu, G., Tumor Microenvironment-Responsive Ultrasmall Nanodrug Generators with Enhanced Tumor Delivery and Penetration. J. Am. Chem. Soc. 2018, 140 (44), 14980-14989.

9. Sun, W.; Luo, L.; Feng, Y.; Qiu, Y.; Shi, C.; Meng, S.; Chen, X.; Chen, H., Gadolinium-Rose Bengal Coordination Polymer Nanodots for MR-/Fluorescence-Image-Guided Radiation and Photodynamic Therapy. Adv. Mater. 2020, 32 (23), 2000377.

10. Xue, C.-C.; Li, M.-H.; Zhao, Y.; Zhou, J.; Hu, Y.; Cai, K.-Y.; Zhao, Y.; Yu, S.-H.; Luo, Z., Tumor microenvironment-activatable Fe-doxorubicin preloaded amorphous $\mathrm{CaCO} 3$ nanoformulation triggers ferroptosis in target tumor cells. Sci. Adv. 2020, 6 (18), eaax1346.

11. Zhu, W.; Zhao, J.; Chen, Q.; Liu, Z., Nanoscale metal-organic frameworks and coordination polymers as theranostic platforms for cancer treatment. Coord. Chem. Rev. 2019, 398, 113009.

12. Poon, C.; He, C.; Liu, D.; Lu, K.; Lin, W., Self-assembled nanoscale coordination polymers carrying oxaliplatin and gemcitabine for synergistic combination therapy of pancreatic cancer. J. Controlled Release 2015, 201, 90-99.

13. He, C.; Liu, D.; Lin, W., Nanomedicine Applications of Hybrid Nanomaterials Built from Metal-Ligand Coordination Bonds: Nanoscale Metal-Organic Frameworks and Nanoscale Coordination Polymers. Chem. Rev. 2015, 115 (19), 11079-11108.

14. Liu, J.; Yang, Y.; Zhu, W.; Yi, X.; Dong, Z.; Xu, X.; Chen, M.; Yang, K.; Lu, G.; Jiang, L.; Liu, Z., Nanoscale metal - organic frameworks for combined photodynamic \& radiation therapy in cancer treatment. Biomaterials 2016, 97, 1-9.

15. Huang, Z.; Huang, L.; Huang, Y.; He, Y.; Sun, X.; Fu, X.; Xu, X.; Wei, G.; Chen, D.; Zhao, C., Phthalocyanine-based coordination polymer nanoparticles for enhanced photodynamic therapy. Nanoscale 2017, 9 (41), 15883-15894.

16. Li, S.; Zou, Q.; Li, Y.; Yuan, C.; Xing, R.; Yan, X., Smart Peptide-Based Supramolecular Photodynamic Metallo-Nanodrugs Designed by Multicomponent Coordination Self-Assembly. J. Am. Chem. Soc. 2018, 140 (34), 10794-10802.

17. Chen, Q.; Wang, X.; Wang, C.; Feng, L.; Li, Y.; Liu, Z., Drug-Induced Self-Assembly of Modified Albumins as Nano-theranostics for Tumor-Targeted Combination Therapy. ACS Nano 2015, 9 (5), 5223-5233.

18. Lovell, J. F.; Jin, C. S.; Huynh, E.; Jin, H.; Kim, C.; Rubinstein, J. L.; Chan, W. C. W.; Cao, W.; Wang, L. V.; Zheng, G., Porphysome nanovesicles generated by porphyrin bilayers for use as multimodal 
biophotonic contrast agents. Nat. Mater. 2011, 10 (4), 324-332.

19. Jin, C. S.; Lovell, J. F.; Chen, J.; Zheng, G., Ablation of Hypoxic Tumors with Dose-Equivalent Photothermal, but Not Photodynamic, Therapy Using a Nanostructured Porphyrin Assembly. ACS Nano 2013, 7 (3), 2541-2550.

20. Liu, M.; Wang, L.; Zheng, X.; Liu, S.; Xie, Z., Hypoxia-Triggered Nanoscale Metal-Organic Frameworks for Enhanced Anticancer Activity. ACS Appl. Mater. Interfaces 2018, 10 (29), 24638-24647.

21. Yang, Y.; Liu, J.; Liang, C.; Feng, L.; Fu, T.; Dong, Z.; Chao, Y.; Li, Y.; Lu, G.; Chen, M.; Liu, Z., Nanoscale Metal-Organic Particles with Rapid Clearance for Magnetic Resonance Imaging-Guided Photothermal Therapy. ACS Nano 2016, 10 (2), 2774-2781.

22. Yang, Y.; Zhu, W.; Dong, Z.; Chao, Y.; Xu, L.; Chen, M.; Liu, Z., 1D Coordination Polymer Nanofibers for Low-Temperature Photothermal Therapy. Adv. Mater. 2017, 29 (40), 1703588.

23. Gelebart, A. H.; Jan Mulder, D.; Varga, M.; Konya, A.; Vantomme, G.; Meijer, E. W.; Selinger, R. L. B.; Broer, D. J., Making waves in a photoactive polymer film. Nature 2017, 546 (7660), 632-636.

24. Aliprandi, A.; Mauro, M.; De Cola, L., Controlling and imaging biomimetic self-assembly. Nat. Chem. 2016, 8 (1), 10-15.

25. Ogi, S.; Sugiyasu, K.; Manna, S.; Samitsu, S.; Takeuchi, M., Living supramolecular polymerization realized through a biomimetic approach. Nat. Chem. 2014, 6 (3), 188-195.

26. Komine, S.; Takahashi, S.; Kojima, T.; Sato, H.; Hiraoka, S., Self-Assembly Processes of OctahedronShaped Pd6L4 Cages. J. Am. Chem. Soc. 2019, 141 (7), 3178-3186.

27. Korevaar, P. A.; George, S. J.; Markvoort, A. J.; Smulders, M. M. J.; Hilbers, P. A. J.; Schenning, A. P. H. J.; De Greef, T. F. A.; Meijer, E. W., Pathway complexity in supramolecular polymerization. Nature 2012, 481 (7382), 492-496.

28. Grantcharova, V.; Alm, E. J.; Baker, D.; Horwich, A. L., Mechanisms of protein folding. Curr. Opin. Struct. Biol. 2001, 11 (1), 70-82.

29. Jonkheijm, P.; van der Schoot, P.; Schenning, A. P. H. J.; Meijer, E. W., Probing the Solvent-Assisted Nucleation Pathway in Chemical Self-Assembly. Science 2006, 313 (5783), 80-83.

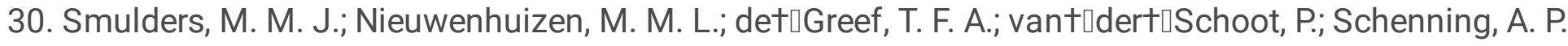
H. J.; Meijer, E. W., How to Distinguish Isodesmic from Cooperative Supramolecular Polymerisation. Chem. - Eur. J. 2010, 16 (1), 362-367.

31. Zhao, Y.; Wang, J.; Pei, R., Micron-Sized Ultrathin Metal-Organic Framework Sheet. J. Am. Chem. Soc. 2020, 142 (23), 10331-10336.

32. Liu, Y.; Wang, H.; Li, S.; Chen, C.; Xu, L.; Huang, P.; Liu, F.; Su, Y.; Qi, M.; Yu, C.; Zhou, Y., In situ supramolecular polymerization-enhanced self-assembly of polymer vesicles for highly efficient photothermal therapy. Nat. Commun. 2020, 11 (1), 1724.

\section{Figures}




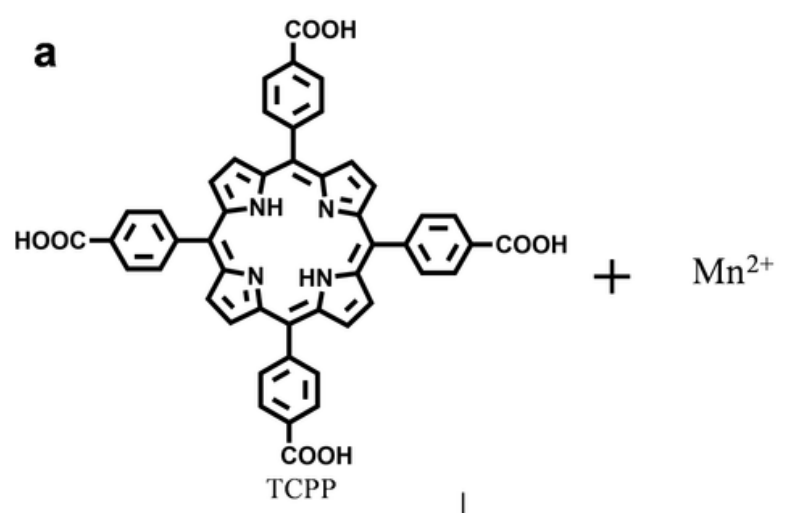

$\Delta \downarrow \mathrm{OH}^{-}$

Mn-TCPP nanosheets

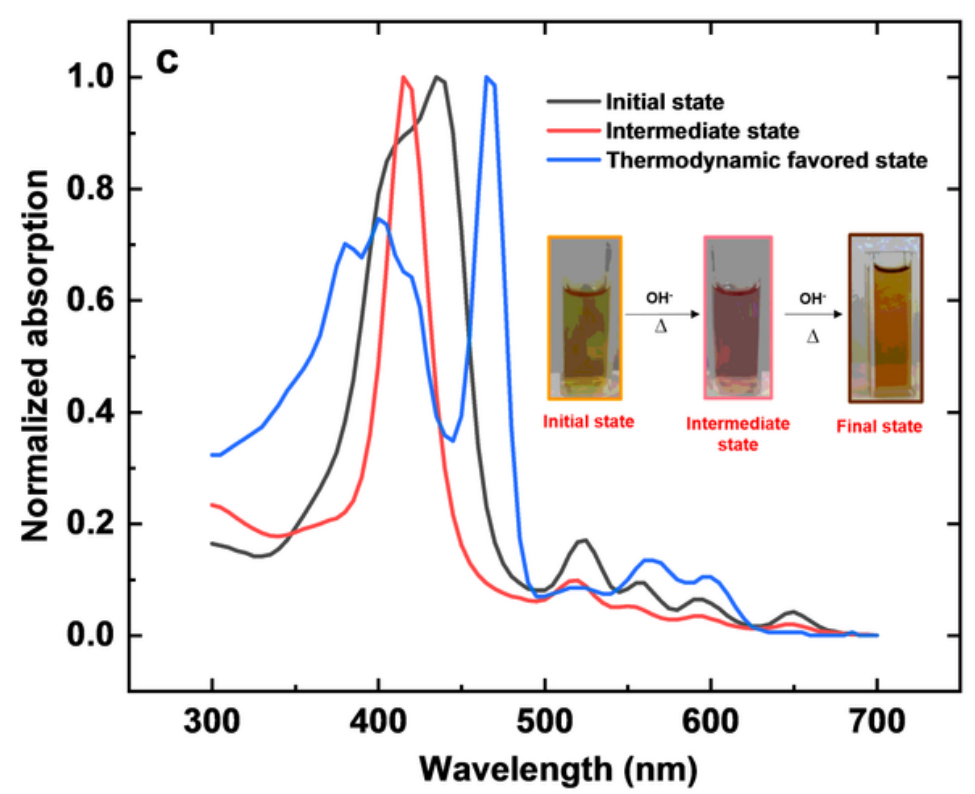

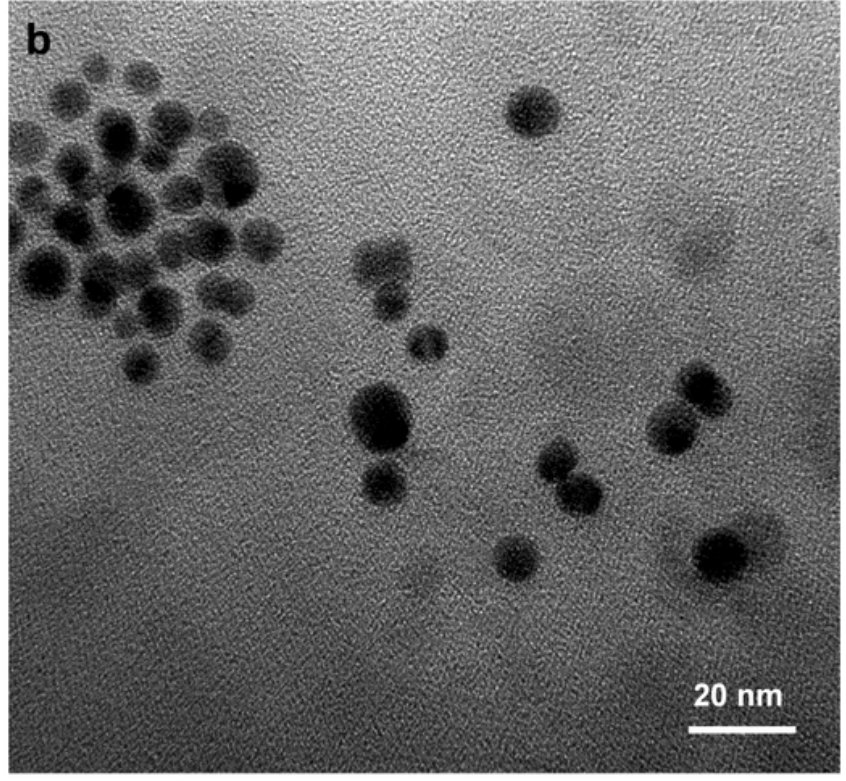

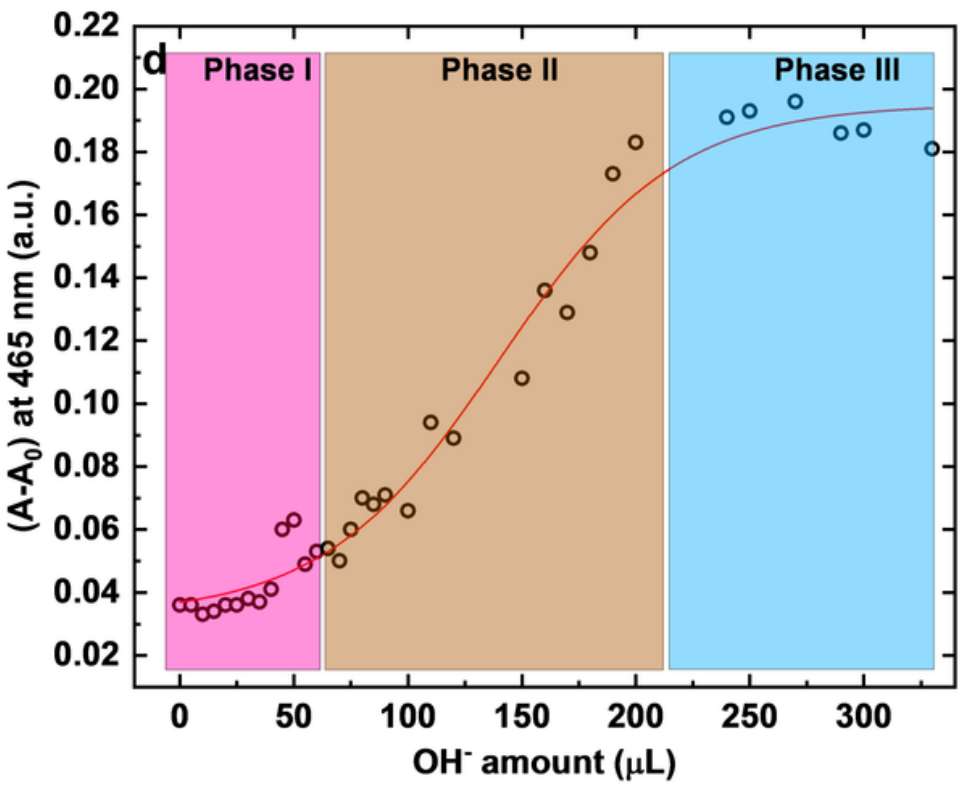

Figure 1

a) Schematic representation of synthesis of Mn-TCPP nanosheets. b) TEM image of Mn-TCPP nanoparticles (thermodynamically favored state). c) Absorption spectra of Mn-TCPP solutions in different states with addition of $\mathrm{OH}-([\mathrm{OH}]=10-2 \mathrm{M})$ : Initial state (black), intermediate state (red), thermodynamic stable state (blue). The insert image is the photography of the solution colors of the corresponding states. d) The absorption intensity changes at $465 \mathrm{~nm}$ as a function of additional $\mathrm{OH}$ amount $([\mathrm{OH}-]=10-2 \mathrm{M})$ at $328 \mathrm{~K}$. 

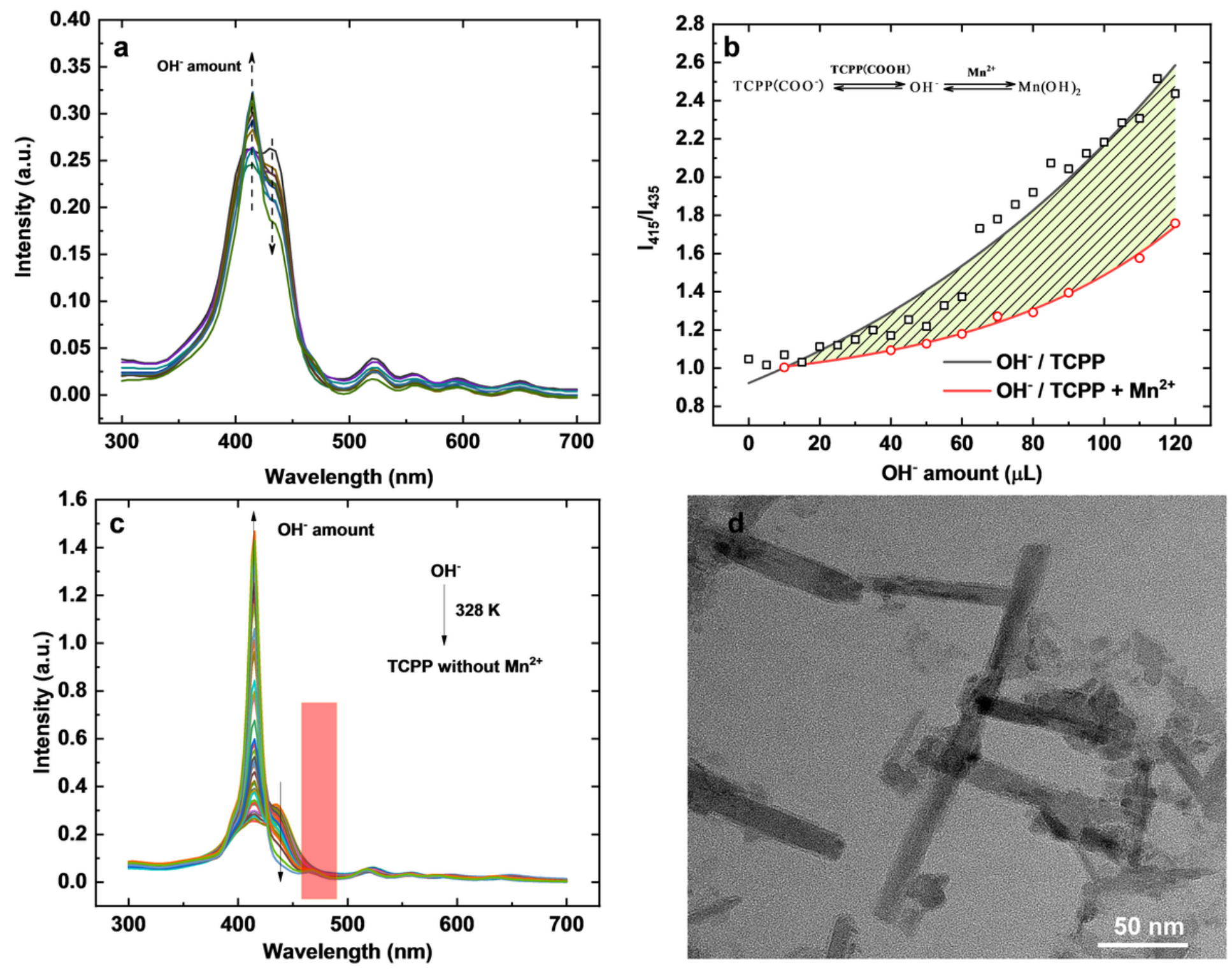

Figure 2

a) UV-vis spectra of Mn/TCPP solutions ([TCPP] $=4.2 \times 10-6 \mathrm{M})$ with addition of $\mathrm{OH}-([\mathrm{OH}-]=10-2 \mathrm{M})$ at $328 \mathrm{~K}$ in the phase I. b) The absorption intensity ratio $1415 / 1435$ changes with addition of $\mathrm{OH}-([\mathrm{OH}-]=10-$ $2 \mathrm{M}$ ) into different solutions: TCPP solutions (black) and TCPP/Mn2+ solutions (red). c) UV-vis spectra of TCPP solutions $(([T C P P]=4.2 \times 10-6 \mathrm{M}))$ with addition of $\mathrm{OH}$ - amount $([\mathrm{OH}-]=10-2 \mathrm{M})$ at $328 \mathrm{~K}$. d) TEM image of intermediate Mn-TCPP aggregates. 

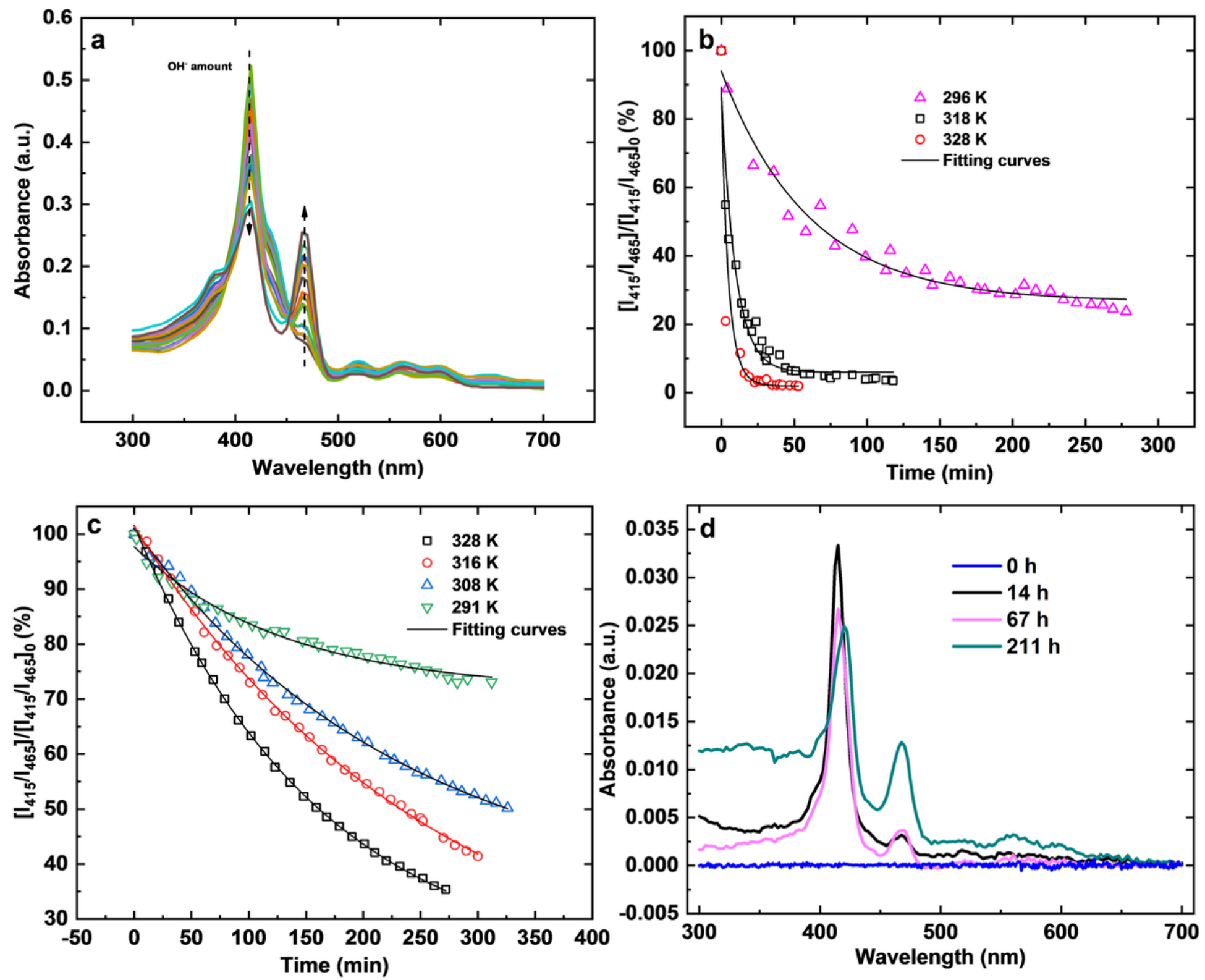

Figure 3

a) UV-vis absorption spectra of Mn-TCPP solutions ([TCPP] $=4.2 \times 10-6 \mathrm{M}$ ) with addition of $\mathrm{OH}$ - at $328 \mathrm{~K}$ in the phase II. Temperature-dependent time course profile (1415/I465) of transformation from intermediate metastable Mn-TCPP aggregates to thermodynamic stable Mn-TCPP aggregates: $b$ ) in the original solution, $c$ ) in deionized water. ([intermediate Mn-TCPP aggregates] $=3.0 \times 10-2 \mathrm{mg} / \mathrm{mL}$ ). d) UVVis absorption spectra of the aqueous solution outside the dialysis bag which was filled with the intermediate Mn-TCPP aggregates at different time. 

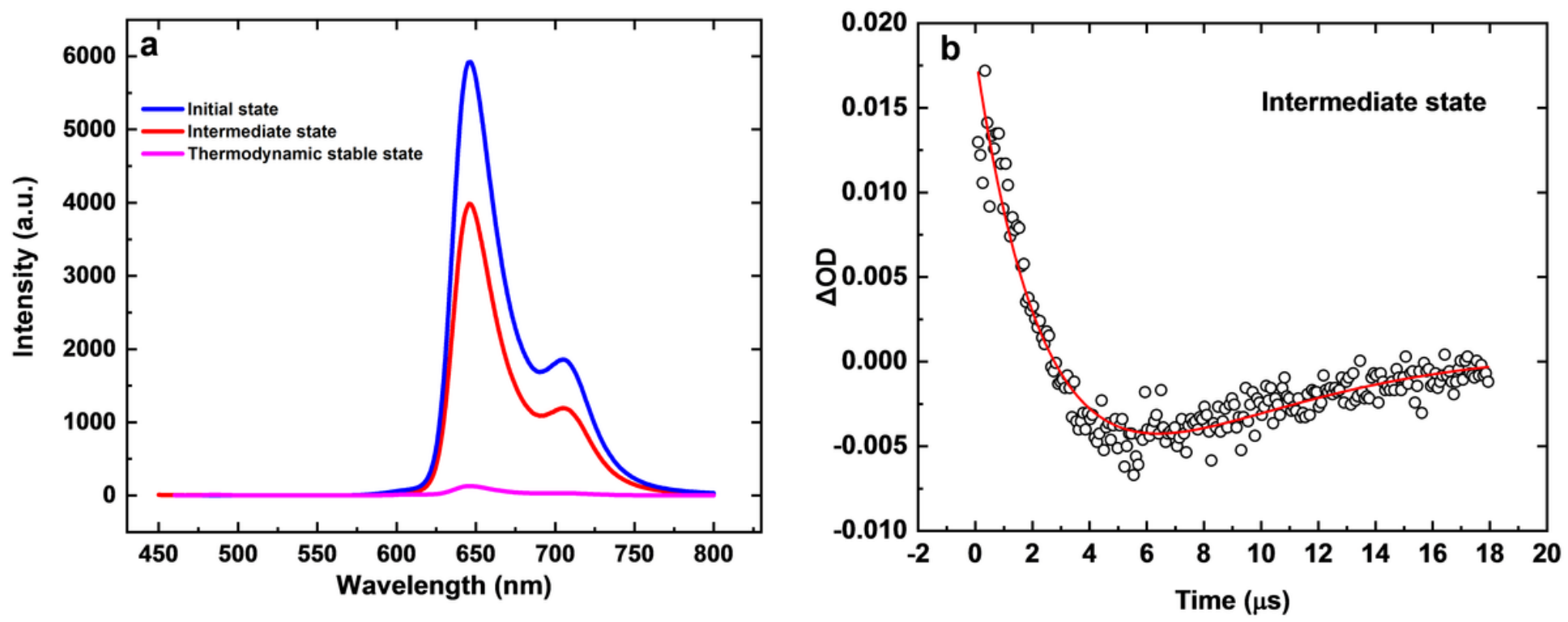

Figure 4

a) FL emission spectra of initial state, intermediate state and thermodynamic stable state during the selfassembly process. b) Transient absorbance spectrum of intermediate Mn-TCPP tape structures in water at $460 \mathrm{~nm}$ under $355 \mathrm{~nm}$ laser pulse. All measurements were performed at room temperature and N2 atmosphere. 


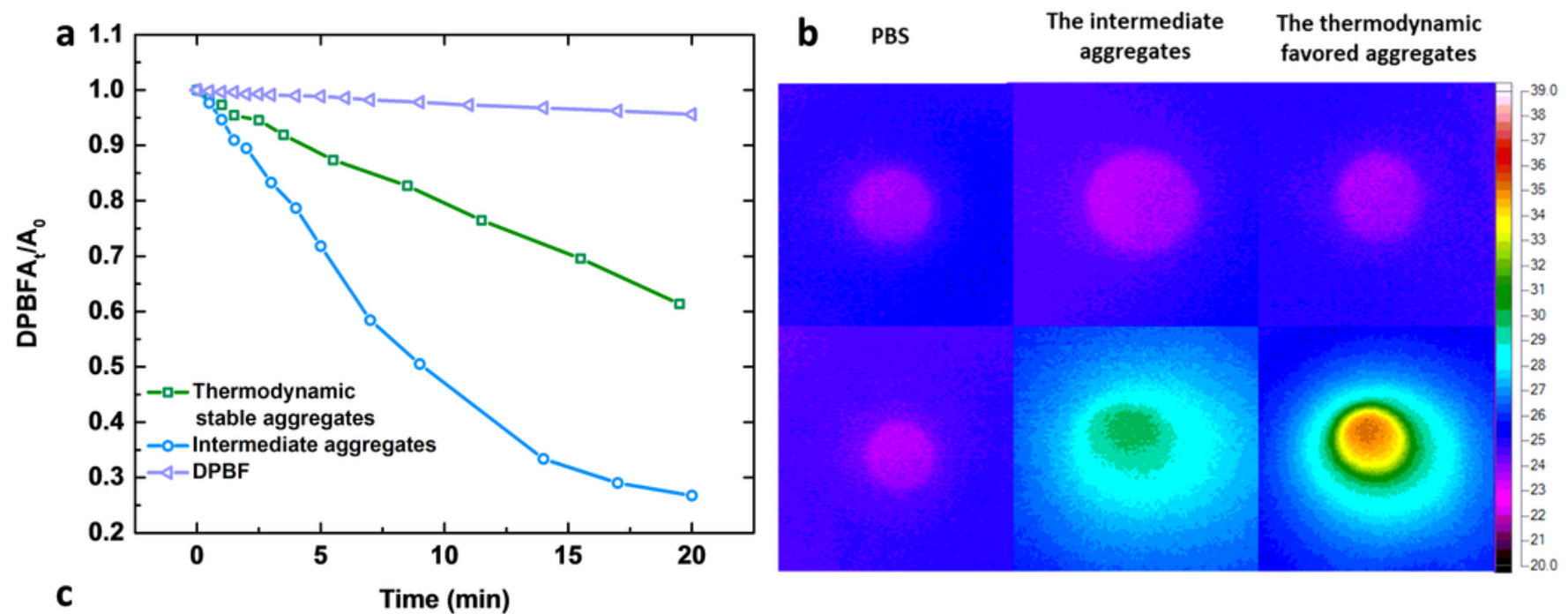

C
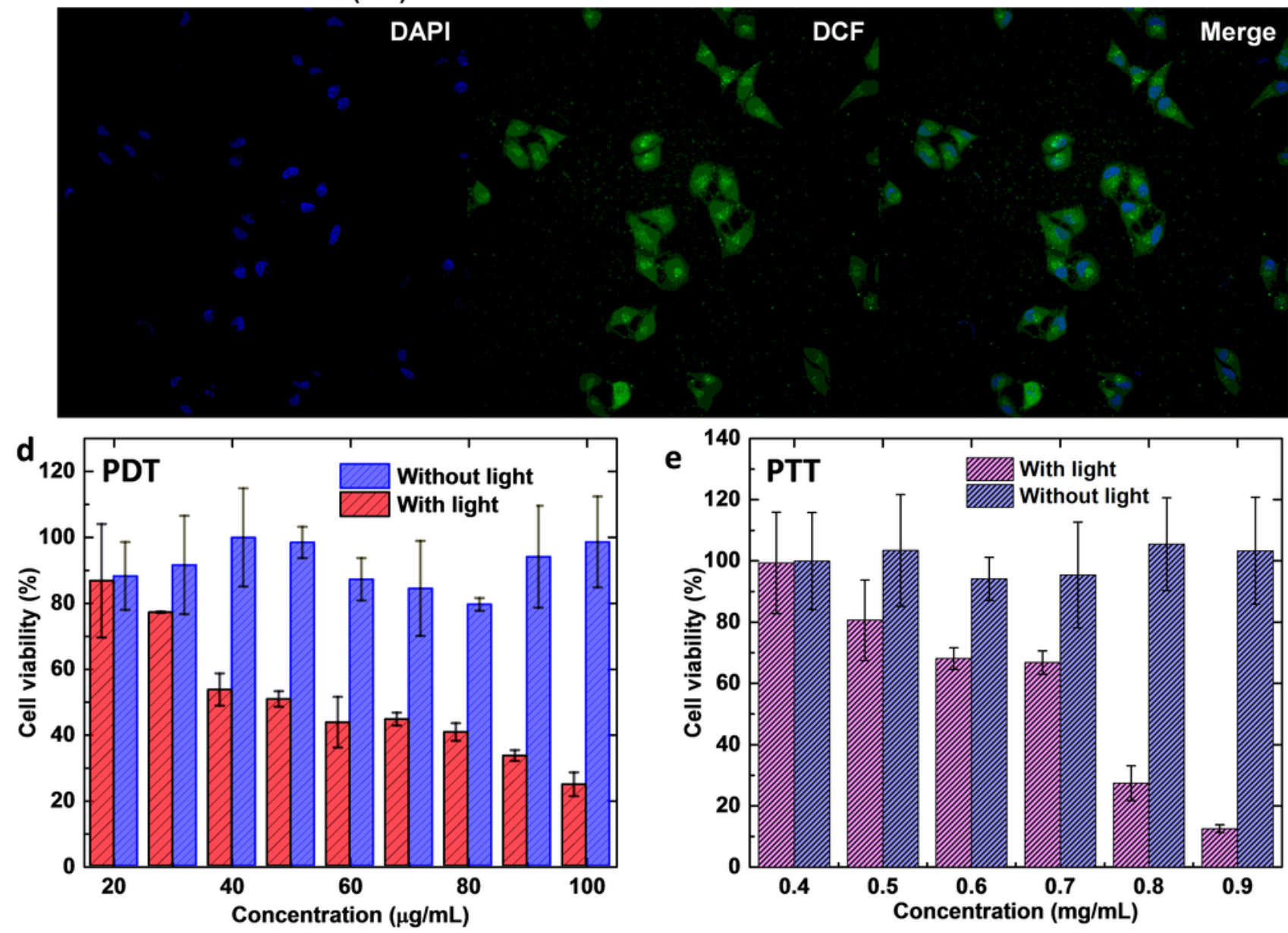

Figure 5

a) 102 production by Mn-TCPP aggregates under LED light irradiation ( $660 \mathrm{~nm}, 12 \mathrm{~mW} \cdot \mathrm{cm}-2)$ using DPBF as the indicator. $b$ ) photothermal photographs of PBS, intermediate Mn-TCPP aggregates (0.76 $\mathrm{mg} / \mathrm{mL}$ ) and thermodynamic stable Mn-TCPP aggregates $(0.84 \mathrm{mg} / \mathrm{mL})$ under laser irradiation $(660 \mathrm{~nm}$, $200 \mathrm{~mW} \cdot \mathrm{cm}-2)$ for $10 \mathrm{~min} . \mathrm{c})$ The CLSM images of HeLa cells after incubation with intermediate MnTCPP aggregates and DCF-DA followed by being stained by DAPI (nucleus). d) Cytotoxicity of Mn-TCPP 
intermediate aggregates against HeLa cells with light irradiation. e) Cell viability test of thermodynamic stable Mn-TCPP aggregates towards HeLa cells under light irradiation. (660 nm, $70 \mathrm{~mW} \cdot \mathrm{cm}-2)$.

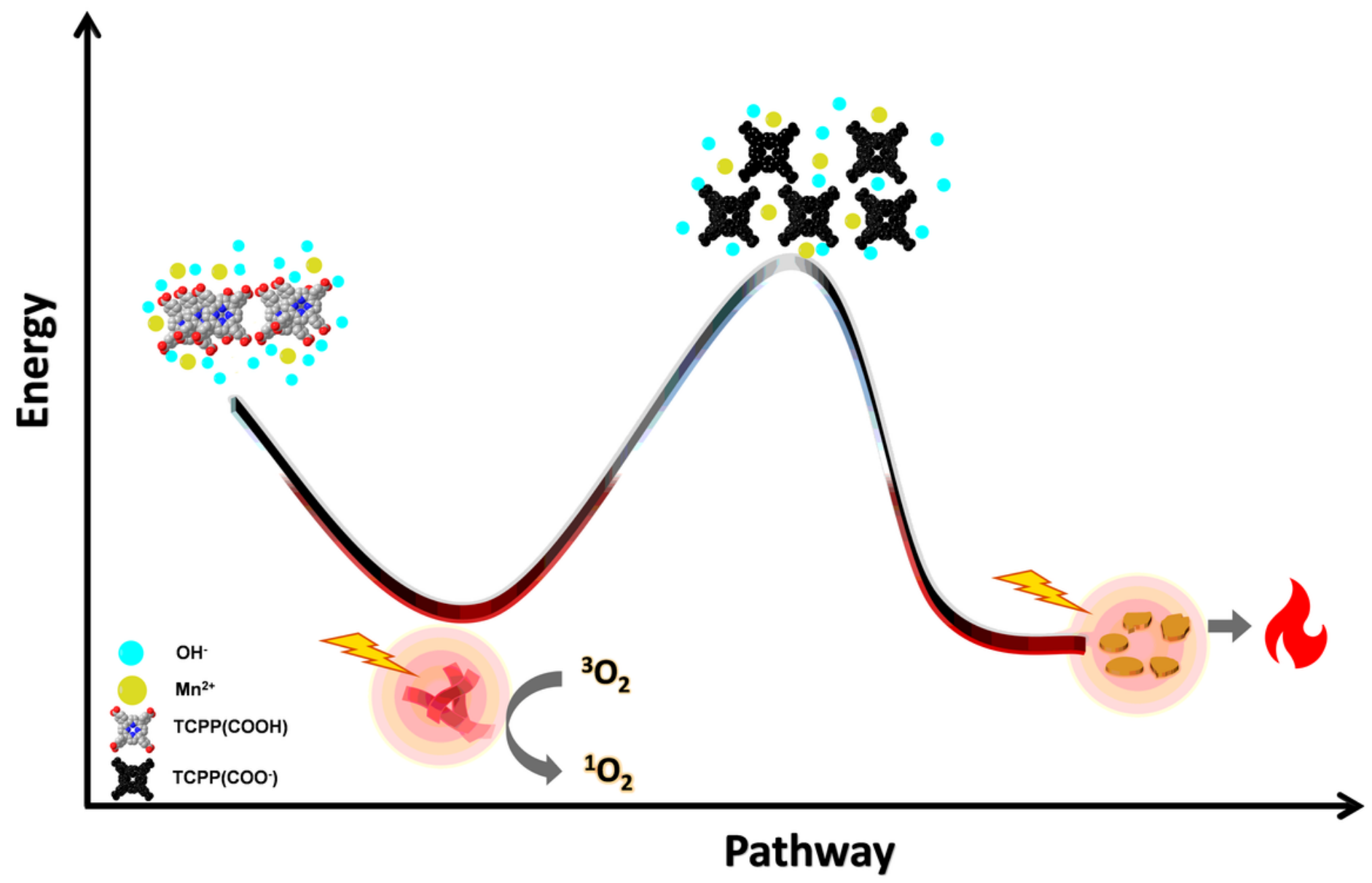

Figure 6

Scheme 1. The schematic presentation of self-assembly pathway of Mn-TCPP nanosheets. 


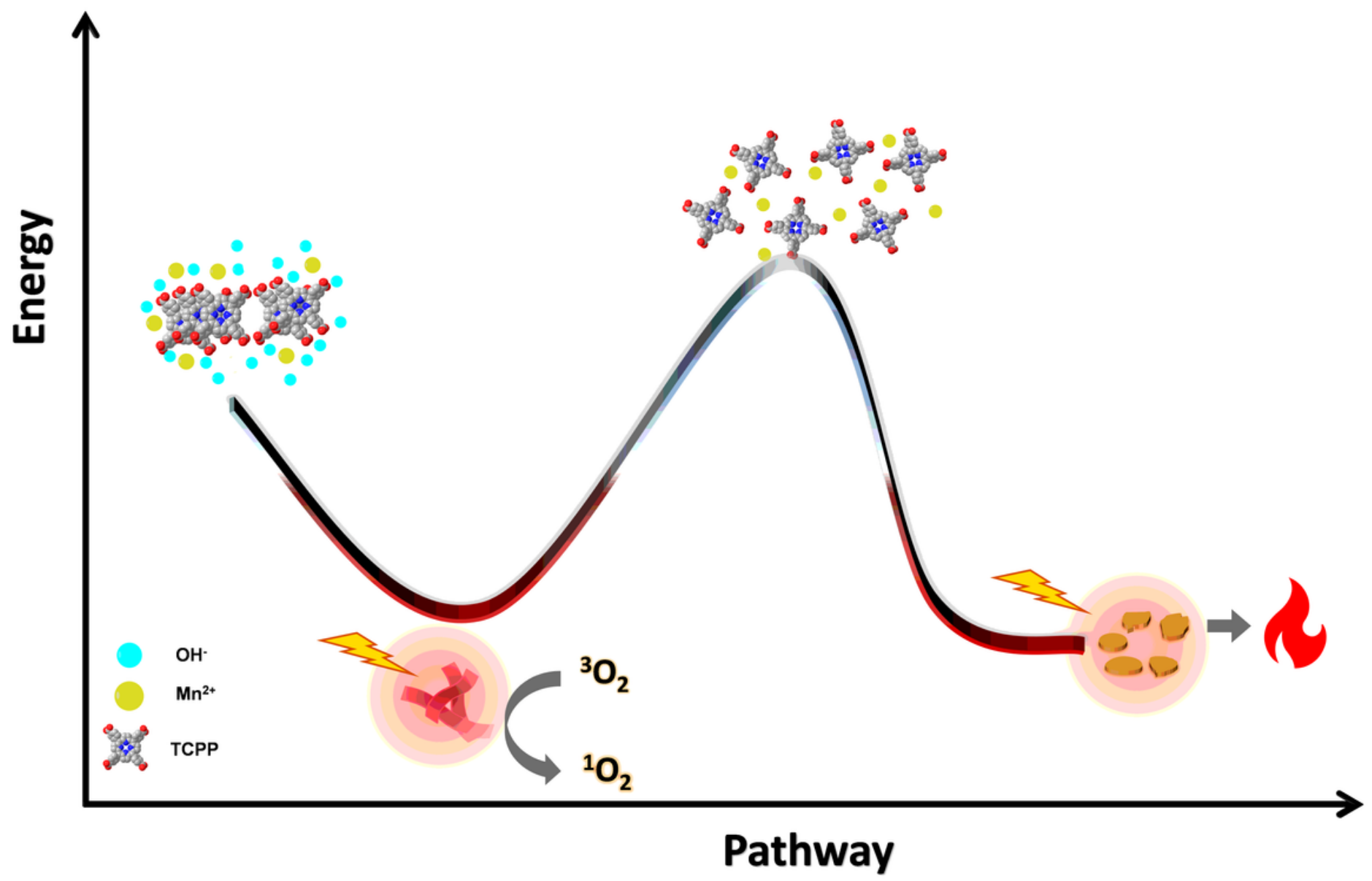

Figure 7

Table of Contents Graphic

\section{Supplementary Files}

This is a list of supplementary files associated with this preprint. Click to download.

- Supplementaryinformation.docx 